\title{
Photometric study of the open cluster NGC 381
}

\author{
Jayanand Maurya, Yogesh Chandra Joshi \\ Aryabhatta Research Institute of Observational Sciences (ARIES), Nainital 263002, India
}

\begin{abstract}
We present $\mathrm{UBVR}_{c} \mathrm{I}_{c}$ photometry of stars, down to the 20th magnitude, in the region of the intermediate-age open cluster NGC 381. The core and the cluster radii are estimated to be $2.99 \pm 0.93$ and $5.6 \pm 0.1$ arcmin, respectively. A total of 350 probable cluster member stars are identified by using proper motion from GAIA as the criterion of membership. The mean proper motion of the cluster is $\bar{\mu}_{x}=1.55 \pm 0.38 \mathrm{mas} \mathrm{yr}^{-1}$,

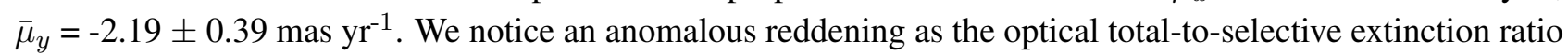
in the direction of the cluster $\mathrm{R}_{\text {cluster }}=3.7 \pm 0.1$. The reddening $\mathrm{E}(\mathrm{B}-\mathrm{V})=0.35 \pm 0.04$ mag is found in the direction of the cluster NGC 381. The distance to the cluster is $957 \pm 93 \mathrm{pc}$ and the cluster has an estimated age of $\log (\mathrm{Age})=8.50 \pm 0.05$ years.
\end{abstract}

Keywords: open cluster: NGC 381 - stars: evolution - techniques: photometric

\section{Introduction}

Open clusters provide an ideal ground for studies of stellar evolution, because all member stars were born from the same molecular cloud, therefore they have approximately the same distance, age, and chemical composition but different masses. This property of open clusters offers constraints on the stellar evolution models and by comparing the colour-magnitude diagram (CMD) and colour-colour diagram (CCD) of star clusters with theoretical evolutionary models, their age, distance and interstellar extinction in the direction of the cluster can be obtained. In order to derive the cluster parameters, the knowledge of membership of the stars is necessary. A proper motion (PM) study is a reliable method for determining the membership of the cluster stars. The study of open star clusters enables us to get a snapshot of stellar evolution of whole populations of stars.

\section{Observation and Data Analysis}

The observations of the open cluster NGC 381 were taken on 21 October 2017 in the Johnson-Cousins $\mathrm{UBVR}_{c} \mathrm{I}_{c}$ filters using the $1.3-\mathrm{m}$ telescope at Devasthal Observatory, India. We found completeness of our data to be beyond $90 \%$ up to magnitude 19 in $\mathrm{U}, 21$ mag in $\mathrm{BVR}_{c}$, and 20 mag in the $\mathrm{I}_{c}$ bands. We compared our present photometry with the previous photometric studies by Phelps et al. (1994) in UBV and Ann et al. (2002) in the UBVI filters. We found that our data are in good agreement with both previous studies except for the U band of Ann et al. (2002). 


\section{Structural properties of the cluster}

\subsection{Radial density profile}

We found the cluster center at the celestial coordinates $(\alpha, \delta)=\left(01^{h} 08^{m} 19.57^{s},+61^{\circ} 35^{\prime} 18.24^{\prime \prime}\right)$ by considering all stars with $\mathrm{V}<18$ mag. The spatial structure and radial density profile (RDP) of the cluster were derived by fitting King (1962) stellar density models as modified by Kaluzny \& Udalski (1992). The methods used are described in Joshi et al. (2012). A $\chi^{2}$ best fit to RDP is also shown in Figure 1. We considered the cluster boundary as the radius value where the star density is $3 \sigma$ above the field star density. We found the core and the cluster radii to be $2.99 \pm 0.93$ and $5.6 \pm 0.1$ arcmin, respectively.

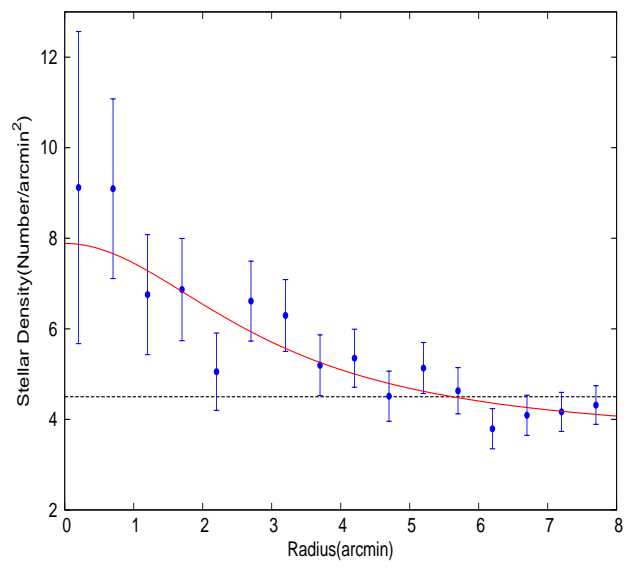

Figure 1: Plot of RDP for NGC 381. The thick continuous (red) line represents the King profile and the horizontal black (dashed) line indicates the field density.

\subsection{Proper motions}

We used the data from the GAIA archive DR2 (Gaia Collaboration et al. 2018) for a proper motion study of the open cluster NGC 381. The PM histogram has a bi-modal distribution as shown in Figure 2(a). The Vector-Point Diagram (VPD) for the NGC 381 region, as shown in Figure 2(b), shows two separate denser regions. After plotting the CMD from stars belonging to these two regions, we found that the small region encircled by the red circle of radius 1 mas $r^{-1}$ corresponds to the cluster members. The center of the circle is determined from the cluster peak in the histogram plots of the PM. The chosen radius is a compromise between losing cluster members and including field stars sharing the cluster mean PM. We found 350 most probable cluster member stars. The mean PM of

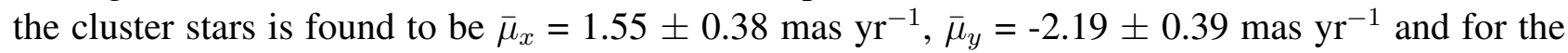
field stars as $\bar{\mu}_{x}=-0.84 \pm 4.13$ mas yr $^{-1}, \bar{\mu}_{y}=-0.47 \pm 2.44$ mas yr$^{-1}$.

(a)

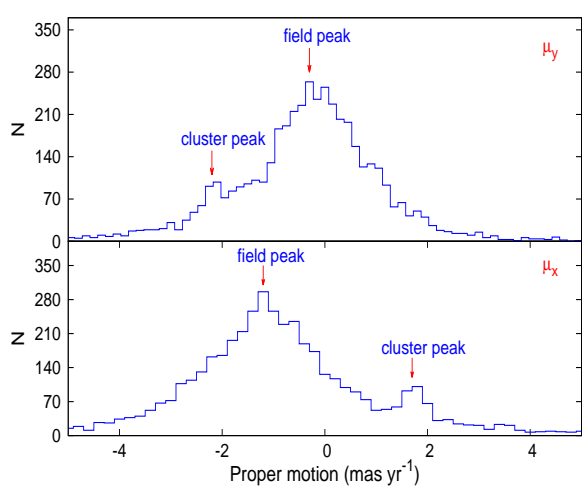

(b)

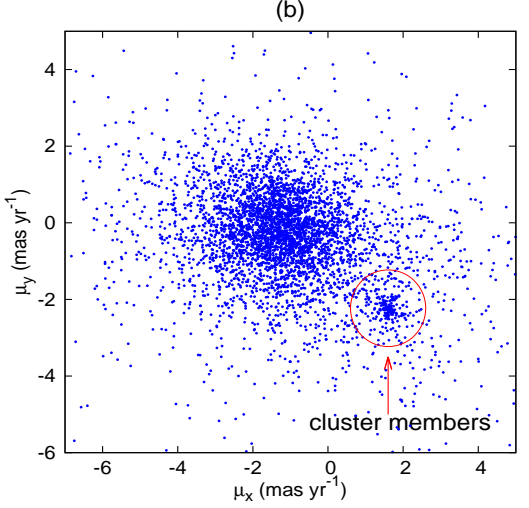

Figure 2: Plots of (a) PM histograms and (b) VPD for NGC 381. The lower panel and upper panel in the left plot represent the histograms of $\mu_{x}$ and $\mu_{y}$, respectively. The cluster members are encircled

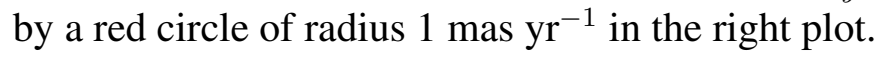




\section{Cluster parameters}

\subsection{The reddening law and colour-colour diagrams}

The normal reddening law, $\mathbf{R}_{v}=\frac{A_{v}}{E(B-V)}$ is not valid for lines of sight that pass through dense clouds (Sneden et al. 1978). The slope of the colour-colour diagram of the form $(\lambda-\mathrm{V}) /(\mathrm{B}-\mathrm{V})$, where $\lambda$ is any broad band filter, distinguishes normal extinction produced by diffused interstellar medium from the extinction caused by abnormal dust grains as shown in Joshi et al. (2014). The plotted CCDs for the present study are shown in Figure 3 where the $\mathrm{J}, \mathrm{H}$, and $\mathrm{K}$ magnitudes are taken from the 2MASS archive (Skrutskie et al. 2006). To derive the value of total-to-selective extinction ratio $\mathrm{R}_{\text {cluster }}$ in the direction of the cluster, we used the approximate relation (Neckel \& Chini 1981)

$$
R_{\text {cluster }}=\frac{m_{\text {cluster }}}{m_{\text {normal }}} \times R_{\text {normal }}
$$

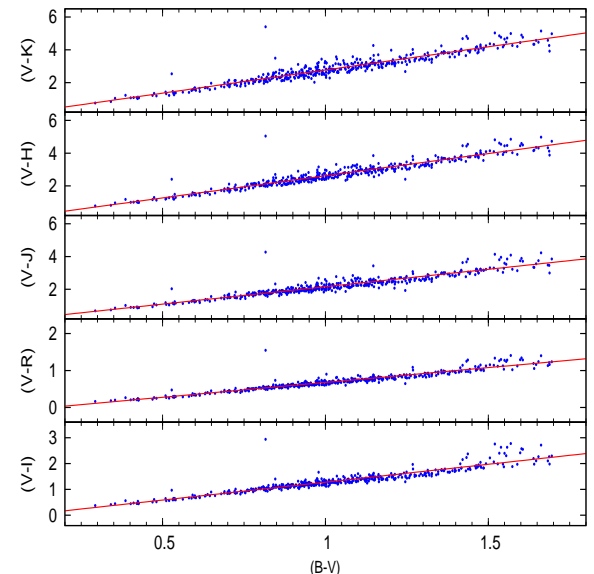

Figure 3: $(\lambda-\mathrm{V}) /(\mathrm{B}-\mathrm{V}) \mathrm{CCDs}$ of NGC 381. The continuous lines represent the best fit slopes.

where, $R_{\text {normal }}=3.1, m_{\text {normal }}$ is the slope of a linear fit to CCDs for normal extinction, and $m_{\text {cluster }}$ is the slope of a linear fit for the cluster stars. By using the above approximation relation, the mean value of $R_{\text {cluster }}$ is calculated to be $3.7 \pm 0.1$ which indicates an anomalous reddening law suggesting a larger dust grain size in the direction of the NGC 381 region.

\subsection{The $\mathrm{E}(\mathrm{B}-\mathrm{V})$ reddening}
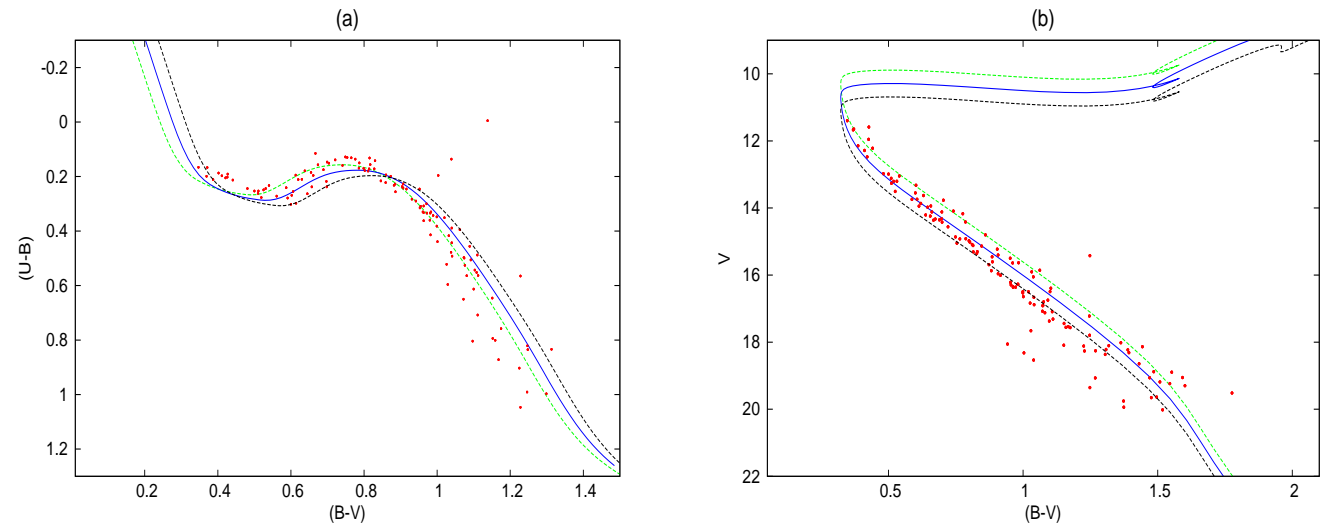

Figure 4: (a) Plots of (a) (U-B)/(B-V) CCD and (b) CMD for NGC 381. The green, blue, and black lines in the left panel correspond to $\mathrm{E}(\mathrm{B}-\mathrm{V})=0.31,0.35$, and $0.39 \mathrm{mag}$, respectively. The green, blue, and black lines in the right panel correspond to the apparent distance modulus $\left(\mathrm{V}-\mathrm{M}_{v}\right)=10.8,11.2$, and 11.6 mag, respectively.

The reddening was determined by using (U-B)/(B-V) CCD constituted from the cluster member stars. We have fitted intrinsic zero-age main-sequence isochrones given by Schmidt-Kaler (1982) to the observed CCD. The CCD along with a best fit isochrone represented by the continuous blue line is shown in Figure 4(a). The two dashed lines drawn at each side of the best-fit isochrone show the 
photometric inaccuracy in the reddening estimation. The best fit was achieved for $\frac{E(B-V)}{E(U-B)}=0.54$ \pm 0.03 and the corresponding value of $\mathrm{E}(\mathrm{B}-\mathrm{V})$ was found to be $0.35 \pm 0.04$ mag in the direction of NGC 381.

\subsection{Distance and Age}

In order to estimate the age and the distance of the cluster, we fitted isochrones published by the Padova group (Marigo et al. 2008) to the CMD of the cluster member stars. The (B-V, V) diagram with fitted isochrone of the solar metallicity $Z=0.019$ is shown in Figure $4(b)$. We chose the solar metallicity because no previous estimation of its metallicity is known for this cluster. The best fit was achieved for $\log ($ Age $)=8.50 \pm 0.05 \mathrm{yr}$, the apparent distance modulus $\left(\mathrm{V}-\mathrm{M}_{v}\right)=11.2 \pm 0.4 \mathrm{mag}$, and the reddening-free distance modulus $\left(\mathrm{V}-\mathrm{M}_{v}\right)_{0}=9.9 \pm 0.4 \mathrm{mag}$ which correspond to an age of $316 \pm 39 \mathrm{Myr}$ and a distance of $957 \pm 93 \mathrm{pc}$ for the cluster NGC 381.

\section{Conclusion}

We present $\mathrm{UBVR}_{c} \mathrm{I}_{c}$ photometric study of the intermediate-age open star cluster NGC 381. The core and the cluster radii are estimated to be $2.99 \pm 0.93$ and $5.6 \pm 0.1$ arcmin, respectively from its radial density profile. We determined the cluster membership of stars using the proper motion criterion and found 350 stars as most probable cluster members. The mean proper motion of the cluster has been

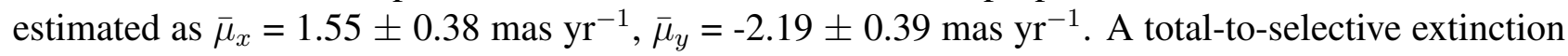
ratio $R_{\text {cluster }}=3.7 \pm 0.1$ was found in the direction of NGC 381 which indicates an anomalous reddening suggesting a larger dust grain size in the cluster region. The derived cluster parameters i.e. the reddening, age, and distance were found to be $\mathrm{E}(\mathrm{B}-\mathrm{V})=0.35 \pm 0.04 \mathrm{mag}, \log (\mathrm{Age})=8.50 \pm$ $0.05 \mathrm{yr}$ and $957 \pm 93 \mathrm{pc}$, respectively. These cluster parameters are in good agreement with earlier studies carried out by Ann et al. (2002) and Phelps et al. (1994), except for the age which is found to be about a factor of three smaller than estimated in the latter case.

\section{References}

Ann H. B., Lee S. H., Sung H. et al. 2002, AJ, 123, 905

Gaia Collaboration, Brown A. G. A., Vallenari A., Prusti T. et al. 2018, A\&A , 616, A1

Joshi Y. C., Joshi S., Kumar B., Mondal S., Balona L. A. 2012, MNRAS, 419, 2379

Joshi Y. C., Balona L. A., Joshi S., Kumar B. 2014, MNRAS, 437, 804

Kaluzny J., Udalski A. 1992, AcA, 42, 29

King I. 1962, AJ, 67, 471

Marigo P., Girardi L., Bressan A. et al. 2008, A\&A, 482, 883

Neckel T., Chini R. 1981, A\&AS, 45, 451

Phelps R. L, Janes K. A. 1994, ApJS, 90, 31

Schmidt-Kaler Th. 1982, in Scaifers K., Voigt H. H., eds, Landolt/Bornstein, Numerical Data and Functional Relationship in Science and Technology, New series, Group VI, Vol. 2b, Springer-Verlag, Berlin, p. 14

Skrutskie M. F., Cutri R. M., Stiening R. et al. 2006, AJ, 131, 1163

Sneden C., Gehrz R. D., Hackwell J. A., York D. G., Snow T. P. 1978, ApJ, 223, 168 